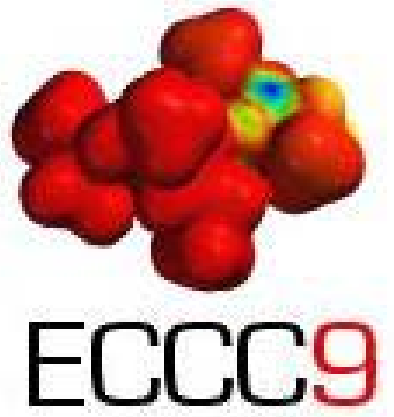

\title{
Theoretical Analysis of the Excited State Properties of Wybutine: A Natural Probe for Transfer RNA Dynamics
}

\section{Ansuman Lahiri ${ }^{1, *}$, Jozef Uličný ${ }^{2}$ and Aatto Laaksonen ${ }^{3}$}

${ }^{1}$ Department of Biophysics, Molecular Biology \& Genetics, University of Calcutta, 92 APC Road, Kolkata 700009, India.

${ }^{2}$ Department of Biophysics, University of P. J. Safarik, Jesenna 5, 04S 54 Košice, Slovak Republic. ${ }^{3}$ Physical Chemistry Division, Arrhenius Laboratory, Stockholm University, S 106 91, Stockholm, Sweden.

*Author to whom correspondence should be addressed. E-mail: alahiri@cubmb.ernet.in

Received: 30 April 2003 / Accepted: 10 October 2003 / Published: 20 February 2004

\begin{abstract}
We have theoretically characterized the ground state and the excited state properties of wybutine, a naturally fluorescent modified base occuring in tRNAs, using configuration interaction singles (CIS) and time dependent density functional (TDDFT) methods. Both gas phase excited state properties and solvent effects, modelled through Onsager reaction field method, were considered. In addition to vertical excitation energies, the fluorescence transitions were calculated, based on $S_{1}$ equilibrium geometry optimized at CIS level. Our computations show encouraging agreement with known experimental data either directly (TDDFT) or after applying empirical scaling (CIS). The fluorescence Stokes' shift for the $S_{0} \leftarrow S_{1}$ transition is computed taking into account the contributions from both intramolecular and solvent reorganization processes. The results suggest that intramolecular relaxation of the $S_{1}$ state accounts for the major part of the magnitude of the Stokes' shift, while the role of solvent reorganization seems to be of less importance.
\end{abstract}

Keywords: CIS, TDDFT, modified base, reaction field; fluorescence lifetime. 


\section{Introduction}

Dynamics of transfer RNAs and particularly of their anticodon domain have been of interest to investigators due to its relevance to codon binding. A number of them has exploited the fluorescence properties of wybutine (Y, fig. 1), a modified base $3^{\prime}$ to the anticodon mGAA of yeast $\mathrm{RNA}^{\text {Phe }}$ to extract information about the intriguing possibility that codon binding may lead to a triggering of a conformational transition which propagates through the entire tRNA molecule thereby exerting a controlling effect on its functions.

Bases in the analogous position of most other tRNAs of known sequence have been shown to be modified adenines. The $\mathrm{Y}$ base differs from all of these bases because its absorption maximum occurs at about $315 \mathrm{~nm}$ [1]. This is considerably to the red of the maxima of most known nucleic acid bases. Significant changes in fluorescence intensities and anisotropy decay rates have been observed when $\mathrm{Mg}^{2+}$ concentration has been changed [2,3]. A $250 \%$ increase in fluorescence intensity occurs gradually as the $\mathrm{Mg}^{2+}$ ion concentration is raised [4]. However, the increase in intensity occurs with virtually no change in the shape of the excitation spectrum and with a small shift in frequency.

If yeast tRNA ${ }^{\text {Phe }}$ is treated at $\mathrm{pH} 2.8$ for $3 \mathrm{hr}$, the $\mathrm{Y}$ base gets specifically cleaved from the tRNA liberating a fluorescent fragment $\mathrm{Y}^{+}$, with very similar emission properties [1]. The fluorescence of $\mathrm{Y}^{+}$ was found to be independent of the solution concentration of $\mathrm{Mg}^{2+}$.

Measurement of the relative efficiency of $\mathrm{Y}^{+}$fluorescence in $\mathrm{H}_{2} \mathrm{O}$ and $\mathrm{CHCl}_{3}$ indicated that $\mathrm{Y}^{+}$ was more than 6 times as fluorescent in a relatively nonpolar solvent than it is in water. The emission maximum of $\mathrm{Y}^{+}$in water occurs at least $20 \mathrm{~nm}$ to the red of the maximum in $\mathrm{CHCl}_{3}$. The emission of $\mathrm{Y}^{+}$in $\mathrm{CHCl}_{3}$, in fact, closely resembles the emission of $\mathrm{Y}$ in intact tRNA ${ }^{\text {Phe }}$.

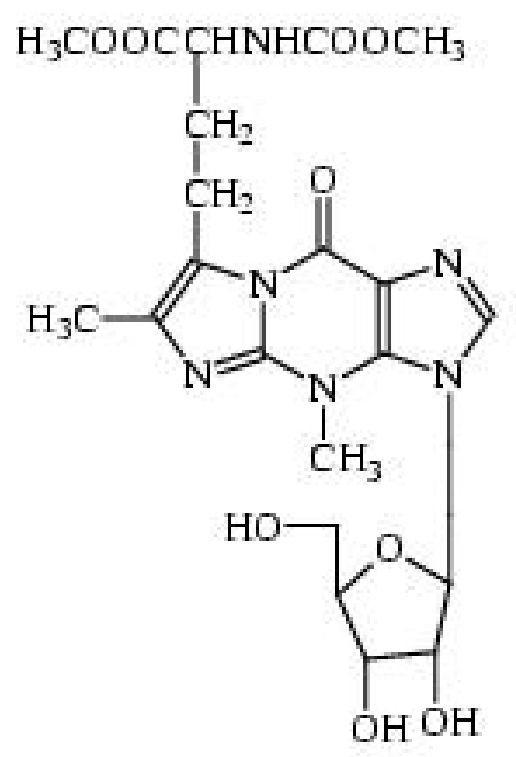

Figure 1. The Y base.

Changes in fluorescence properties of wybutine have also been observed on codon binding $[3,5,6]$. These results indicate the existence of at least two distinct conformations [6] or three [3] which differ in their solvation environments and mobilities. One of these probable conformations, with the least amount of mobility, is postulated to be that observed in the crystal structure, with Y37 nicely stacked in between A36 and A38, thereby encountering a predominantly hydrophobic environment. The other (or other two) 


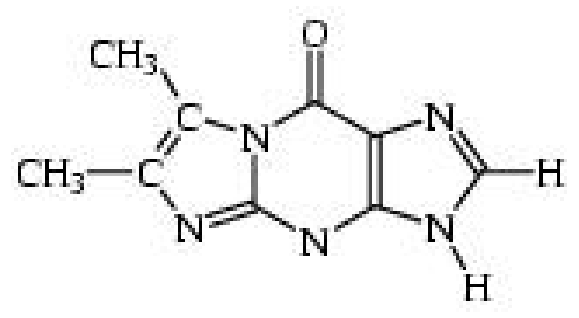

Figure 2. The wybutine model used in the present calculation.

substates which arise at low magnesium concentrations or with codon binding are characterized by larger mobilities and is postulated to arise due to unstacking of the Y37 base either due to destabilisation of the anticodon loop or due to interaction with the codon bases.

We recently carried out extensive molecular dynamics simulations of the solvated anticodon domain of yeast tRNA ${ }^{\text {Phe }}$ in the presence and absence of the codon trinucleotides UUC and UUU complexed to the anticodon bases [7]. We found from this simulation that binding of codon does indeed cause a significant structural deviation which is localized near the wybutine region. Wybutine also showed instances of increased fluctuation with codon binding.

To our knowledge, there is as yet no theoretical investigation of the fluorescence properties of wybutine although a CNDO/S estimate of the ground and excited state properties and energies of wybutine and its substructures was previously reported [8].

Here we report results obtained from the application of semi-empirical, CIS and TDDFT methods to calculate the excited state properties of wybutine and compare them to previous theoretical and experimental results. The ground and excited state properties were studied both in the gas phase and the water phase using the simplified Onsager reaction field method. In the present work we have studied a simplified model of wybutine in which its long tail has been replaced by a methyl group. This preserves the essential electronic properties while reducing the computational efforts considerably. For free form purines, interpretation of the electronic spectra is complicated by the $\mathrm{N}_{7} \mathrm{H} \leftrightarrow \mathrm{N}_{9} \mathrm{H}$ tautomeric equilibrium. Since binding of wybutine to the sugar-phosphate backbone atoms occurs through $\mathrm{N}_{9}$, we have considered only the $\mathrm{N}_{9} \mathrm{H}$ form for this study. We plan to consider the effect of $\mathrm{Mg}^{2+}$, explicit water molecules and stacking of adjacent bases in subsequent works.

\section{Computational Details}

The base $\mathrm{Y}$ has been observed in slightly different forms: $\mathrm{Y}$ in yeast tRNA ${ }^{\text {Phe }}[9]$; Yw in wheat germ tRNA $^{\text {Phe }}[10]$ and Yt in Torulopsis utilis tRNA ${ }^{\text {Phe }}[11]$.

The initial structure for $\mathrm{Y}$ base for subsequent calculations was obtained from a $2.7 \AA$ resolution yeast tRNA $^{\text {Phe }}$ crystal structure [12] from the Brookhaven database (PDB code 6TNA). Structure of a wybutine-derivative is also obtainable in the CMBI database [13] and structure of the Yt base from [14].

The truncated model base used in our calculations is shown in figure 2 where a hydrogen and a methyl group have been manually added to the $\mathrm{N} 9$ and $\mathrm{C} 12$ positions to replace the sugar and the long chain respectively.

The semi-empirical calculations in the present work has been carried out with MOPAC 7.0 [15]. The truncated structure was BFGS optimized with a gradient norm requirement of 0.01 using a restricted Hartree-Fock AM1 hamiltonian [16]. The excited state calculation was performed using a multielec- 
Table 1. Transition energies in $\mathrm{eV}[\mathrm{nm}]$ and oscillator strengths at ground state geometry $\left(S_{0}\right)$. The CIS energies were scaled by a factor of 0.72 .

\begin{tabular}{ccccc}
\hline & AM1 & CIS/6-31G(d,p) & TDDFT/6-31G(d,p) & Experiment \\
\hline$S_{0} \rightarrow S_{1}$ & $4.16[298]$ & $4.67[265]$ & $4.23[293]$ & $3.96[313]$ \\
$f$ & - & 0.09 & 0.06 & - \\
\hline
\end{tabular}

tron configuration interaction procedure. For calculating the $S_{0} \rightarrow S_{1}$ transition, 9 configurations were included. Using a similar procedure, the $S_{1}$ state was optimized and the energy gaps calculated.

$A b$ initio results were obtained with Gaussian98 suite of programs [17] using the configuration interaction singles (CIS) and the time dependent density functional (TDDFT) procedures. Configuration interaction singles method has been described as the simplest level of theory that can be used to include some correlation effects through the mixing of excited determinants [18]. Recently, time dependent density-functional theory has been applied also with success for calculating the excitation energies of large molecules [19].

To properly account for orbital relaxation effects, permanent dipole moments of the excited state were computed using the CIS density. Geometry optimisations of the $S_{1}$ state were carried out at the CIS/6$31 \mathrm{G}(\mathrm{d}, \mathrm{p})$ level as well as the CIS/6-311G(d,p) level. The excited state properties were also calculated in the gas phase at the optimized geometries with TDDFT method using the B3LYP functional [20]. The ground state geometry was optimized from the initial structure using a HF/6-311G(d,p) procedure.

Solvation effects on the absorption and emission transitions of wybutine were calculated using the Onsager model [21], which considers the dipolar interaction of a spherical cavity containing the solute dipole with the reaction field of a dielectric continuum, characterized by its refractive index and static dielectric constant. The cavity radius was taken to be $4.83 \AA$ for both the ground and the excited state. The dielectric constant was taken to be 78.39. Calculations for fluorescence Stokes' shift in water was at the CIS/6-31G(d,p) level where the $S_{1}$ state geometry was optimized at the CIS/6-311G(d,p) level.

\section{Results and Discussion}

\section{Transition characteristics}

Spectroscopic studies on Y base and related compounds reveal the existence of three major transitions above $220 \mathrm{~nm}$. The $S_{0} \rightarrow S_{1}$ transition is observed as a weak transition at $313 \mathrm{~nm}$ followed by another weak transition at $263 \mathrm{~nm}$ and a strong transition at $235 \mathrm{~nm}$ [9].

The previous CNDO/S computation of the spectral features of wybutine showed only a partial agreement with the observed features [8]. The $S_{0} \rightarrow S_{1}$ transition was computed to lie at higher energies (283 $\mathrm{nm}$ ) than observed although the weak nature of the transition was reproduced. The second calculated transition $\left(S_{0} \rightarrow S_{2}\right)$ had so weak intensity, that it was not expected to be observed. Accordingly, the next observed transition was ascribed to the $S_{0} \rightarrow S_{3}$ excitation. But the intensity of the transition was calculated to be much higher than what was observed. The energy of the $T_{1} \rightarrow S_{0}$ was found to be closer to the experimental value. Calculation on the emission characteristics of the molecule was not reported.

A preliminary semi-empirical calculation with the AM1 hamiltonian on our model base and using the multi-electron configuration interaction (as implemented in MOPAC 7.0) showed an approximate agreement with the experimental values (tables 1 and 2). 
Table 2. Transition energies in $\mathrm{eV}[\mathrm{nm}]$ and oscillator strengths at the excited state geometry $\left(S_{1}\right)$. The CIS energies have been scaled by a factor of 0.72 . The third and fourth columns correspond to geometry optimisation at CIS/6-31G(d,p) level while the fifth and sixth columns correspond to geometry optimisation at CIS/6-311G(d,p) level

\begin{tabular}{cllllll}
\hline & AM1 & CIS/6- & TDDFT/6- & CIS/6- & TDDFT/6- & Experiment \\
& & $31 \mathrm{G}(\mathrm{d}, \mathrm{p})$ & $31 \mathrm{G}(\mathrm{d}, \mathrm{p})$ & $31 \mathrm{G}(\mathrm{d}, \mathrm{p})$ & $31 \mathrm{G}(\mathrm{d}, \mathrm{p})$ & \\
\hline$S_{0} \rightarrow S_{1}$ & $3.06[405]$ & $4.11[302]$ & $3.95[314]$ & $3.02[411]$ & $2.99[414]$ & $2.76[450]$ \\
$f$ & - & 0.17 & 0.07 & 0.20 & 0.11 & - \\
\hline
\end{tabular}

Semi-empirical AM1 calculations for the $S_{0} \rightarrow S_{1}$ transitions and its reverse yielded the transition wavelengths of $298 \mathrm{~nm}$ and $405 \mathrm{~nm}$ respectively. Experimentally, the emission maximum of Y base in tRNA in solution is observed to occur around $450 \mathrm{~nm}$. It is interesting that AM1 reproduces the observed values much better than ab initio CIS calculations and is comparable to TDDFT results for these transitions.

As a rule, excitation energies are substantially overestimated in the CIS calculations, since it fails to properly account for differences in dynamical electron correlation in the ground and the excited states. Broo and Holmén have suggested that this difference is comparable in all the nucleobases and have used a scaling factor of 0.72 for the excitation energies of $\pi-\pi^{*}$ transitions [22].

In table 1 we report the transition energies at the ground state geometry and in table 2 the energetics for excited state $\left(S_{1}\right)$ geometries optimized at the CIS/6-31G(d,p) and CIS/6-311G(d,p) levels respectively. The time-dependent density functional results are seen to approximate better to the experimental values in the ground state. However, in the higher level opimized excited state geometries, the CIS results were comparable to the DFT energies after scaling.

\section{Solvation effects on the absorption and emission spectra}

Solvent dependent spectral shifts usually arise from either general or specific solvent effects. The former results from interactions of the dipole moment of the fluorescent species with the reaction field in the surrounding solvent. Specific effects, on the other hand, result from short range interactions with solvent molecules like hydrogen bonding.

The CNDO/S calculation of the changes in electron densities on excitation of the Y base showed that both imidazole rings were involved and that a considerable amount of charge transfer occured [8].

Spectral shift is largely influenced by the dipole change in going from the ground state to the excited state. However, for wybutine we found the dipole change not to be so large. For a representative calculation within the self consistent reaction field model, it was observed to change from 8.6 D in the ground state to $9.5 \mathrm{D}$ in the excited state (table 3). However, on subsequent reorganisation of the excited state obtained by optimisation, it relaxes to 7.1 D. In comparison, Stark effect experiments on tryptophan has yielded a dipole change of $6 \mathrm{D}$ [23].

The direction of the dipole moment vector (figure 3) did not change appreciably for the absorption process. However, in the emission process, a large change was observed concomitant to a nonplanar distorted shape for the excited state optimized structure (table 3).

Within the framework of the Onsager model, the solvation energy is estimated following [24] by considering the interaction of a spherical cavity of radius $a_{0}$ containing the solute with the reaction field 


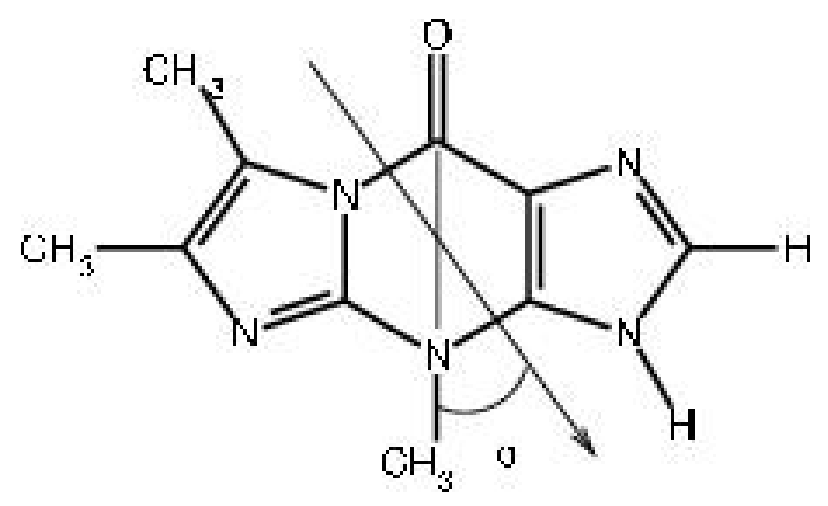

Figure 3. A scheme for describing the angle between the electric dipole moment vector and the molecular reference axis in the molecular plane.

of the solvent.

In absorption, the ground state is in equilibrium with the solvent and the reaction field is given by $R=\left(2 \mu_{0} / a_{0}^{3}\right)\{(\epsilon-1) /(2 \epsilon+1)\}$, where $\epsilon$ is the static dielectric constant of the solvent. The solvation energy of the ground state is thus

$$
E_{0}^{s o l v}=-\frac{2}{a_{0}^{3}} \mu_{0} \cdot \mu_{0}\left\{\frac{\epsilon-1}{2 \epsilon+1}\right\}
$$

The vertical transition is to an $S_{1}$ state in which only the electronic response is realized. The reaction field felt by the final state arising from the orientational part of solvent polarisation corresponds to the fully relaxed ground state. The solvation energy of the $S_{1}$ state in the absorptive process is thus given by $[24]$

$$
E_{1}^{\text {solv }}=-\frac{2}{a_{0}^{3}}\left[\mu_{1} \cdot \mu_{0}\left\{\frac{\epsilon-1}{2 \epsilon+1}-\frac{n^{2}-1}{2 n^{2}+1}\right\}+\mu_{1} \cdot \mu_{1}\left\{\frac{n^{2}-1}{2 n^{2}+1}\right\}\right]
$$

where $n$ is the solvent refractive index. The two terms on the right hand side of equation 2 reflect the contributions from the orientational and electronic solvent responses, respectively. The vertical $S_{0} \rightarrow S_{1}$ excitation energy in the solution phase is

$$
E_{0 \rightarrow 1}^{s o l}=E_{0 \rightarrow 1}^{g a s}+E_{1}^{s o l v}-E_{0}^{s o l v}
$$

Table 3. $S_{0}$ and $S_{1}$ dipole moments, solvation energies and vertical transition energies (energies are in $\mathrm{eV})$. The emission data are from the CIS/6-311G(d,p) optimized structure.

\begin{tabular}{cccccc}
\hline State & $\mu / \mathrm{D}$ & $\phi$ & $E^{\text {Solv }}$ & $E_{0 \rightarrow 1}^{\text {gas }}$ & $E_{0 \rightarrow 1}^{\text {soln }}$ \\
\hline Absorption & & & & & \\
$\mathrm{S}_{0}$ & 8.5745 & 18.0 & -0.40 & 4.65 & $4.59[270]$ \\
$\mathrm{S}_{1}$ & 9.5074 & 18.4 & -0.46 & & \\
Emission & & & & & \\
$\mathrm{S}_{0}$ & 7.9505 & 28.5 & -0.34 & 3.02 & $3.07[404]$ \\
$\mathrm{S}_{1}$ & 7.1479 & 11.4 & -0.29 & & \\
\hline
\end{tabular}


In the emission process the situation is reversed. Assuming that the solvent relaxation time is much shorter than the $S_{1}$ lifetime, the excited state is fully relaxed and the ground state experiences the orientational reaction field corresponding to the dipole moment of the $S_{1}$ state.

In table 3 we show the solvation energies for the $S_{0}$ and $S_{1}$ states obtained using the dipole moments corresponding to the ground state and the excited state optimized structures. The static dielectric constant and the refractive index for these calculations were taken as $\epsilon=78.39$ and $n=1.33$.

From the values for the $S_{0} \rightarrow S_{1}$ and $S_{1} \rightarrow S_{0}$ transition energies in table 3, the fluorescence Stokes' shift in water is calculated as $134 \mathrm{~nm}$, which compares favourably with the experimental value of $\sim 130$ $\mathrm{nm}$. The contribution from the solvent reorganisation energy is seen to be pretty small however. This agrees with similar calculations on aminopurine [24].

\section{Conclusion}

We have reported here results from calculation of electronic transition energies and other transition characteristics of the hypemodified wybutine base which is utilized as a naturally occuring fluorescence probe for conformational dynamics of yeast tRNA ${ }^{\text {Phe }}$. The calculations were carried out at semi-empirical level using the AM1 hamiltonian and at ab initio level using CIS and TDDFT methods. Both gas phase and solution properties were investigated.

When compared to experimental observations, our results suggest that AM1 at the semi-empirical level and TDDFT at the ab initio level better reproduces the transition energies than the CIS method. However, although CIS was worse in giving transition energies, the Stokes' shift in water calculated with CIS showed a good agreement with observations. We also found that the spectral shift of wybutine in an aqueous environment arises from vibrational relaxation rather than solvent reorganisation.

\section{Acknowledgement}

Support from the Swedish Natural Science Research Council (NFR) is gratefully acknowledged.

\section{References}

1. Thiebe, R.; Zachau, H. A specific modification next to the anticodon of phenylalanine transfer ribonucleic acid. Eur. J. Biochem. 1968, 5, 546-555.

2. Ehrlich, R.; Lefevre, J.-F.; Remy, P. Fluorimetric study of the complex between yeast phenylalanine-trna synthetase and tRNA ${ }^{\text {Phe }}$. Eur. J. Biochem. 1980, 103, 145-153.

3. Claesens, F.; Rigler, R. Conformational dynamics of the anticodon loop in yeast tRNA ${ }^{\text {Phe }}$ as sensed by the fluorescence of wybutine. Eur. Biophys. J. 1986, 13, 331-342.

4. Beardsley, K.; Tao, T.; Cantor, C. Studies on the conformation of the anticodon loop of phenylalanine transfer ribonucleic acid. Effect of environment on the fluorescence of Y base. Biochemistry 1970, $9,3524-3532$.

5. Eisinger, J.; Feuer, B.; Yamane, T. Luminescence and binding studies on tRNA-phe. Proc. Natl. Acad. Sci. USA 1970, 65, 638-644. 
6. Labuda, D.; Porschke, D. Magnesium ion inner sphere complex in the anticodon loop of phenylalanine transfer ribonucleic acid. Biochemistry 1982, 21, 49-53.

7. Lahiri, A.; Nilsson, L. Molecular dynamics of the anticodon domain of yeast tRNA ${ }^{\text {Phe }}$ : Codonanticodon interaction. Biophys. J. 2000, 79, 2276-2289.

8. Evleth, E. M.; Lerner, D. A. Theoretical analysis of the photophysical properties of $\epsilon$-adenine and the RNA ${ }^{\text {Phe }}$ y-base. Photochem. Photobiol. 1977, 26, 103-108.

9. Nakanishi, K.; Furutachi, N.; Funamizu, M.; Grunberger, D.; Weinstein, I. Structure of the fluorescent Y base from yeast phenylalanine transfer ribonucleic acid. J. Am. Chem. Soc. 1970, 92, 7617-7619.

10. Dudock, B. S.; Katz, G.; Taylor. E. K.; Holley, R. W. Primary structure of wheat germ phenylalanine transfer RNA. Proc. Natl. Acad. Sci. USA 1969, 62, 941-945.

11. Kasai, H.; Goto, M.; Ikeda, K.; Zama, M.; M.Y. Structure of wye (Yt base) and wyosine (Yt) from torulopsis utilis phenylalanine transfer ribonucleic acid. Biochemistry 1976, 15, 898-904.

12. Sussman, J. L.; Holbrook, S. R.; Warrant, R. W.; Kim, S. H. Crystal structure of yeast phenylalanine transfer RNA. I. crystallographic refinement. J. Mol. Biol. 1978, 123, 607-630.

13. Itaya, T.; Watanabe, N.; Mizutani, A. Synthesis of optically active forms of hydroxy-Y base, the minor componenet of rat liver phenylalanine transfer ribonucleic acid. Tetrahedron Lett. 1986, 27, 4043-4046.

14. Nygjerd, G.; McAlister, J.; Sundaralingam, M. The molecular and crystal structure of the fluorescent base Yt (1h-4,6-dimethylimidazo[1,2-a]purine-9-one) in tRNA. Acta Crsyt. 1975, B31, 413-417.

15. Stewart, J. J. P. Mopac 7.0. a general molecular orbital package; QCPE, 1993, 688.

16. Dewar, M. J. S.; Zoebisch, E. G.; Healy, E. F.; Stewart, J. J. P. AM1: A new general purpose quantum mechanical model. J. Am. Chem. Soc. 1985, 107, 3902-3909.

17. Frisch, M. J.; Trucks, G. W.; Schlegel, H. B.; Scuseria, G. E.; Robb, M. A.; Cheeseman, J. R.; Zakrzewski, V. G.; Montgomery, J. A.; Stratmann, R. E.; Burant, J. C.; Dapprich, S.; Millam, J. M.; Daniels, A. D.; Kudin, K. N.; Strain, M. C.; Farkas, O.; Tomasi, J.; Barone, V.; Cossi, M.; Cammi, B.; Mennucci, B.; Pomelli, C.; Adamo, C.; Clifford, S.; Ochterski, J.; Petersson, G. A.; Ayala, P. A.;Cui, K.; Morokuma, K.; Malick, D. K.; Rabuck, A. D.; Raghavachari, K.; Foresman, J. B.; Cioslowski, J.; Ortiz, J. V.; Stefanov, B. B.; Liu, G.; Liashenko, A.; Piskorz, P.; Komaromi, I.; Gomperts, R.; Martin, R. L.; Fox, D. J.; Keith, T.; Al-Laham, M. A.; Peng, C. Y.; Nanayakkara, A.; Gonzalez, C.; Challacombe, M.; Gill, P. M. W.; Johnson, B. G.; Chen, W.; Wong, M. W.; Andres, J. L.; Head-Gordon, M.; Replogle, E. S.; Pople, J. A. Gaussian 98 (Revision A.7); Gaussian Inc., Pittsburgh PA, 1998.

18. Foresman, J. B.; Head-Gordon, M.; Pople, M. J.; Frisch, J. A. Toward a systematic molecular orbital theory for excited states. J. Phys. Chem. 1992, 96, 135-149. 
19. Stratmann, R. E.; Scuseria, G. E.; Frisch, M. J. An efficient implementation of time-dependent density-functional theory for the calculation of excitation energies of large molecules. J. Chem. Phys. 1998, 109, 8218-8224.

20. Becke, A. D. Density-functional thermochemistry. III. the role of exact exchange. J. Chem. Phys. 1993, $98,5648-5652$.

21. Onsager, L. Electric moments of molecules in liquids. J. Am. Chem. Soc. 1936, 58, 1486-1493.

22. Broo, A.; Holmén, A. Calculations and characterization of the electronic spectra of DNA bases based on ab initio MP2 geometries of different tautomeric forms. J. Phys. Chem. 1997, 101, 35893600 .

23. Pierce, D.; Boxer, S. Stark effect spectroscopy of tryptophan. Biophys. J. 1995, 68, 1583-1591.

24. Jean, J. M.; Hall, K. B. Theoretical study of the excited state properties and transitions of 2aminopurine in the gas phase and in solution. J. Phys. Chem. A 2000, 104, 1930-1937.

(C) 2004 by MDPI (http://www.mdpi.net). Reproduction for noncommercial purposes permitted. 